\title{
A metric theorem for restricted Diophantine approximation in positive characteristic
}

\author{
by \\ Simon Kristensen (Edinburgh and Aarhus)
}

To Maurice Dodson on his retirement

1. Introduction. Let $\mathbb{F}$ be the finite field with $k=p^{l}$ elements, let $\mathbb{F}[X]$ denote the ring of polynomials with coefficients from $\mathbb{F}$, let $\mathbb{F}(X)$ denote the field of fractions over this ring and let $\mathbb{F}\left(\left(X^{-1}\right)\right)$ denote the field of formal Laurent series with coefficients from $\mathbb{F}$, i.e.,

$$
\mathbb{F}\left(\left(X^{-1}\right)\right)=\left\{\sum_{i=n}^{\infty} a_{i} X^{-i}: n \in \mathbb{Z}, a_{i} \in \mathbb{F}\right\} .
$$

We define a non-Archimedean absolute value on $\mathbb{F}\left(\left(X^{-1}\right)\right)$ by

$$
\left|\sum_{i=n}^{\infty} a_{i} X^{-i}\right|= \begin{cases}0 & \text { whenever } a_{i}=0 \text { for all } i \in \mathbb{Z}, \\ k^{-n} & \text { whenever } a_{n} \neq 0 \text { and } a_{i}=0 \text { for } i<n .\end{cases}
$$

We can interpret $\mathbb{F}\left(\left(X^{-1}\right)\right)$ as the completion of $\mathbb{F}(X)$ with respect to this absolute value. Note that in addition to the usual non-Archimedean property of the absolute value, $|f+g| \leq \max \{|f|,|g|\}$, we also have

$$
|f| \neq|g| \Rightarrow|f+g|=\max \{|f|,|g|\} \text {. }
$$

Diophantine approximation in $\mathbb{F}\left(\left(X^{-1}\right)\right)$, where a generic element is approximated by elements from the field of fractions $\mathbb{F}(X)$, has been studied by numerous authors (the survey papers $[9,14]$ contain some of the known results). Broadly speaking, the object of study has been variations over inequalities of the form

$$
|f-P / Q|<\psi(|Q|),
$$

where $f \in \mathbb{F}\left(\left(X^{-1}\right)\right)$ and $P, Q \in \mathbb{F}[X]$ with $Q \neq 0$. The study of the metric theory of Diophantine approximation in this setting, in which the Haar mea-

2000 Mathematics Subject Classification: 11J83, 11J61.

The author is a William Gordon Seggie Brown Fellow. 
sure and Hausdorff dimension of sets arising from such inequalities are studied, was begun by de Mathan in [10], who proved an analogue of Khinchin's theorem in Diophantine approximation. The author extended this theorem to systems of linear forms [8].

Let $m, n \in \mathbb{N}$ and $\psi: \mathbb{F}[X]^{m} \rightarrow\left\{k^{r}: r \in \mathbb{Z}\right\}$ be some function. Let $S \subseteq \mathbb{F}[X]^{m}$. For $\mathbf{v} \in \mathbb{F}\left(\left(X^{-1}\right)\right)^{n}$, let $\|\mathbf{v}\|$ denote the distance from $\mathbf{v}$ to the nearest element in $\mathbb{F}[X]^{n}$ with respect to the metric induced by the norm $|\mathbf{x}|_{\infty}=\max \left\{\left|x_{1}\right|, \ldots,\left|x_{n}\right|\right\}$, where $\mathbf{x}=\left(x_{1}, \ldots, x_{n}\right) \in \mathbb{F}\left(\left(X^{-1}\right)\right)^{n}$. In this paper, we study the set

$$
\begin{aligned}
\mathcal{W}_{S}(m, n ; \psi)=\left\{A \in \operatorname{Mat}_{m \times n}\left(\mathbb{F}\left(\left(X^{-1}\right)\right)\right):\right. & \|\mathbf{q} A\|<\psi(\mathbf{q}), \\
& \text { for infinitely many } \mathbf{q} \in S\},
\end{aligned}
$$

where $\operatorname{Mat}_{m \times n}\left(\mathbb{F}\left(\left(X^{-1}\right)\right)\right)$ denotes the space of $m$ by $n$ matrices $A$ with coefficients from $\mathbb{F}\left(\left(X^{-1}\right)\right)$ and $\mathbf{q} A$ denotes the usual matrix product.

Recently, Inoue and Nakada proved a Khinchin type theorem for the special case $\mathcal{W}_{Q}(1,1 ; \psi)[7$, Theorem 1]. Namely, they showed that the Haar measure of $\mathcal{W}_{Q}(1,1 ; \psi)$ is null or full according as the series $\sum_{q \in S} \psi(q)$ converges or diverges, under the additional assumptions that the approximating fractions $P / Q$ are on lowest terms, that $S$ is of the form $\left\{q \in \mathbb{F}[X]:|q|=k^{d}\right.$, $\left.d \in S^{\prime}\right\}$ for some subset $S^{\prime} \subseteq \mathbb{N}$ and that $\psi(q)$ depends only on $|q|$.

In the real case, the Hausdorff dimension of the analogous sets for $m=1$ and arbitrary $n$ in the special case when $\psi=|q|^{-v}$ was determined by Borosh and Fraenkel [2]. Various more general cases were studied using the notion of ubiquitous systems by Rynne [12]. This was subsequently extended to an even more general form of approximation by Dickinson and Rynne [4].

We will consider the analogue of the case originally considered by Rynne [12]. It is the purpose of the present paper to determine the Hausdorff dimension - and in some cases the Haar measure - of the sets $\mathcal{W}_{S}(m, n ; \psi)$ for $m \geq 2$, subject to very mild constraints on the approximation function $\psi$. We will denote by $\mu$ the Haar measure on $\mathbb{F}\left(\left(X^{-1}\right)\right)$, normalised so that the unit ball,

$$
U=\left\{f \in \mathbb{F}\left(\left(X^{-1}\right)\right):|f|<1\right\} \subseteq \mathbb{F}\left(\left(X^{-1}\right)\right),
$$

has measure 1 . By abuse of notation, the normalised Haar measure on vector spaces $V$ over $\mathbb{F}\left(\left(X^{-1}\right)\right)$ will also be denoted by $\mu$. Similarly, we will denote by $U$ the unit cube in $V$, i.e., the $\operatorname{dim}(V)$-fold Cartesian product of $U$ by itself. This should cause no confusion.

We need a few definitions. First, we need an appropriate notion of the exponent of convergence for the sequence $S$ given by

$$
v(S)=\inf \left\{v \in \mathbb{R}: \sum_{\mathbf{q} \in S}|\mathbf{q}|_{\infty}^{-v}<\infty\right\} .
$$

We also need the appropriate notion of the order at infinity $\lambda(\psi)$ of the 
function $1 / \psi$,

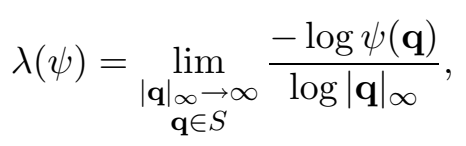

defined whenever the limit exists. We can now state the first main theorem.

Theorem 1. Let $\psi: \mathbb{F}[X]^{m} \rightarrow\left\{k^{r}: r \in \mathbb{Z}\right\}$. Suppose that $\psi(\mathbf{q})$ depends only on $|\mathbf{q}|_{\infty}$, is decreasing as a function of $|\mathbf{q}|_{\infty}$, and that the order at infinity of the function $\psi$ exists.

(i) If $n \lambda(\psi)<v(S)$, then $\mathcal{W}_{S}(m, n ; \psi)$ is full with respect to the Haar measure on $\operatorname{Mat}_{m \times n}\left(\mathbb{F}\left(\left(X^{-1}\right)\right)\right)$.

(ii) If $n \lambda(\psi) \geq v(S)$, then

$$
\operatorname{dim}_{\mathrm{H}}\left(\mathcal{W}_{S}(m, n ; \psi)\right)=n(m-1)+\frac{n+v(S)}{1+\lambda(\psi)},
$$

where $\operatorname{dim}_{\mathrm{H}}(E)$ denotes the Hausdorff dimension of the set $E$.

Note that while we can calculate the dimension for each value of $v(S)$ and $\lambda(\psi)$, we are not able to show in general whether the measure of $\mathcal{W}_{S}(m, n ; \psi)$ is null or full for the critical value $n \lambda(\psi)=v(S)$ with the methods of the present paper.

In analogy with a result of Rynne [13], we may deduce from Theorem 1 the Hausdorff dimension of the set $\mathcal{W}_{S}(m, n ; \psi)$ with very mild conditions on $\psi$. Indeed, it suffices to assume that $\psi$ is bounded, non-negative, and that $\psi(\mathbf{q})>0$ for infinitely many $\mathbf{q}$. Note that in this case by defining

$$
\widehat{\psi}(\mathbf{q})= \begin{cases}\psi(\mathbf{q}) & \text { whenever } \mathbf{q} \in S, \\ 0 & \text { otherwise }\end{cases}
$$

we would have $\mathcal{W}_{S}(m, n ; \psi)=\mathcal{W}_{\mathbb{F}[X]^{m}}(m, n ; \widehat{\psi})$. Consequently, for such more general $\psi$, we omit the set $S$ from our notation and talk about $\mathcal{W}(m, n ; \psi)$. We define

$$
\eta(\psi)=\inf \left\{\eta \in \mathbb{R}: \sum_{\mathbf{q} \in \mathbb{F}[X]^{m}}|\mathbf{q}|_{\infty}^{n}\left(\frac{\psi(\mathbf{q})}{|\mathbf{q}|_{\infty}}\right)^{\eta}<\infty\right\} .
$$

Theorem 2. Suppose that $\psi: \mathbb{F}[X]^{m} \rightarrow\left\{k^{r}: r \in \mathbb{Z}\right\} \cup\{0\}$ is bounded, non-negative, and that $\psi(\mathbf{q})>0$ for infinitely many $\mathbf{q}$. Then

$$
\operatorname{dim}_{\mathrm{H}}(\mathcal{W}(m, n ; \psi))=n(m-1)+\min \{\eta(\psi), n\} .
$$

Theorem 2 generalises part (i) of Theorem 1. As before, an optimal condition for when the measure is full does not follow from our approach. This is however a very difficult problem generalising the Duffin-Schaeffer conjecture (see, e.g., [15]) to systems of linear forms over $\mathbb{F}\left(\left(X^{-1}\right)\right)$. For simultaneous 
approximation, such a result is known for reals [11] and has been announced for formal power series [6].

2. Proof of Theorem 1. We need to prove three things. First, we will show that the right hand side in (ii) is an upper bound on the Hausdorff dimension of $\mathcal{W}_{S}(m, n ; \psi)$. Note that this implies that the Haar measure is zero when $n \lambda(\psi)>v(S)$. Subsequently, we need to show that the measure is full in case (i) and that the right hand side in (ii) is also a lower bound on the dimension.

In the following, matrices in $\operatorname{Mat}_{m \times n}\left(\mathbb{F}\left(\left(X^{-1}\right)\right)\right)$ will be identified with vectors in $\mathbb{F}\left(\left(X^{-1}\right)\right)^{m n}$. Given a vector $\mathbf{x}$ and a set $V$, we will denote by $\operatorname{dist}(\mathbf{x}, V)$ the minimal distance from $\mathbf{x}$ to $V$ in the absolute value $|\cdot|_{\infty}$. Given two positive real quantities $a, b$ we will use the Vinogradov notation and say that $a \ll b$ if $a=O(b)$ in Landau's $O$-notation, i.e., if there is a constant $K>0$ such that $a \leq K b$. Of course, when the notation is used, $a$ and $b$ will be functions of some parameter, and the implied constant $K$ will be independent of this parameter. If $a \ll b$ and $b \ll a$, we will write $a \asymp b$.

2.1. An upper bound. We note that the set $\mathcal{W}_{S}(m, n ; \psi)$ is invariant under translations by elements from $\operatorname{Mat}_{m \times n}(\mathbb{F}[X])$. Hence, we restrict ourselves to considering the intersection of $\mathcal{W}_{S}(m, n ; \psi)$ and the unit cube $U$. We will prove that the upper bound is the right one when $n=1$. In this case, we are determining the dimension of the set

$$
\mathcal{W}_{S}^{*}(m, 1 ; \psi)=\left\{A \in U:\|\mathbf{q} A\|<\psi\left(|\mathbf{q}|_{\infty}\right) \text { for infinitely many } \mathbf{q} \in S\right\} .
$$

We omit the details of the case $n>1$ for ease of notation, but give an outline of the differences from the one-dimensional case at the end of this part of the proof.

Consider the $(m-1)$-dimensional affine subspaces for which $\|\mathbf{q} A\|=0$, i.e., the affine subspaces

$$
H(\mathbf{q}, p)=\{A \in U: \mathbf{q} A=p\} .
$$

Note that for this set to be non-empty, we must have $|p| \leq|\mathbf{q}|_{\infty}$. Clearly, points $A$ satisfying the inequality $|\mathbf{q} A-p|_{\infty}<\psi\left(|\mathbf{q}|_{\infty}\right)$ for a fixed $p$ and $\mathbf{q}$ must lie within $\psi\left(|\mathbf{q}|_{\infty}\right)|\mathbf{q}|_{\infty}^{-1}$ of $H(\mathbf{q}, p)$.

Let $\varepsilon>0$ be arbitrary. By definition, for $|\mathbf{q}|_{\infty}$ large enough, $\psi\left(|\mathbf{q}|_{\infty}\right) \leq$ $|\mathbf{q}|_{\infty}^{-\lambda(\psi)+\varepsilon}$. Suppose that $|\mathbf{q}|_{\infty}$ is large enough for this to hold.

We cover the neighbourhoods of $H(\mathbf{q}, p)$ by $\asymp|\mathbf{q}|_{\infty}^{(1+\lambda(\psi)-\varepsilon)(m-1)}$ balls of radius $2|\mathbf{q}|_{\infty}^{-\lambda(\psi)+\varepsilon-1}$. Call this cover $\mathcal{C}(\mathbf{q}, p)$. For any $M>0$, the sets $\bigcup_{|\mathbf{q}|_{\infty} \geq M, \mathbf{q} \in S} \bigcup_{|p| \leq|\mathbf{q}|_{\infty}} \mathcal{C}(\mathbf{q}, p)$ cover $\mathcal{W}_{S}^{*}(m, 1 ; \psi)$. The $s$-length of this cover $\mathcal{C}(M)$ is 


$$
\begin{aligned}
\ell^{s}(\mathcal{C}(M)) & \ll \sum_{|\mathbf{q}|_{\infty} \geq M}^{\substack{\mathbf{q} \in S \\
|p| \leq|\mathbf{q}|_{\infty}}} \sum_{\infty}|\mathbf{q}|_{\infty}^{(1+\lambda(\psi)-\varepsilon)(m-1)}|\mathbf{q}|_{\infty}^{-(\lambda(\psi)-\varepsilon+1) s} \\
& \ll \sum_{\substack{|\mathbf{q}|_{\infty} \geq M \\
\mathbf{q} \in S}}|\mathbf{q}|_{\infty}^{1+(1+\lambda(\psi)-\varepsilon)(m-1)-(\lambda(\psi)-\varepsilon+1) s} \\
& \ll \sum_{\substack{|\mathbf{q}|_{\infty} \geq M \\
\mathbf{q} \in S}}|\mathbf{q}|_{\infty}^{-v(S)-\varepsilon^{\prime}},
\end{aligned}
$$

whenever

$$
s>(m-1)+\frac{1+v(S)}{1+\lambda(\psi)-\varepsilon},
$$

for some $\varepsilon^{\prime}>0$, which tends to zero as $\varepsilon$ does. Hence, we may choose $\varepsilon>0$ (which was arbitrary) such that the last series of (6) tends to zero as $M$ tends to infinity. By the Hausdorff-Cantelli Lemma (see e.g. [1]), the Hausdorff dimension of $\mathcal{W}_{S}^{*}(m, 1 ; \psi)$ must then be less than or equal to the right hand side of (7). As $\varepsilon>0$ was arbitrary, this implies that the upper bound holds.

To prove the statement for $n>1$, we note that the multidimensional analogues of $H(\mathbf{q}, p)$ will be $n(m-1)$-dimensional affine spaces. We may again cover the neighbourhoods by balls of radius $\asymp|\mathbf{q}|_{\infty}^{-\lambda(\psi)+\varepsilon-1}$. Using elementary upper bounds for the $s$-length of the resulting cover implies the result.

2.2. Reduction to simpler $\psi$. We will now show that it suffices to consider the case when $\psi\left(|\mathbf{q}|_{\infty}\right)$ is of the form $|\mathbf{q}|_{\infty}^{-\lambda(\psi)}$. For this, we use again the existence of $\lambda(\psi)$. By (4), for any $\varepsilon>0$ there is an $r_{0} \in S$ such that for any $\mathbf{q} \in S$ with $|\mathbf{q}|_{\infty} \geq k^{r_{0}}$

$$
|\mathbf{q}|_{\infty}^{-\lambda(\psi)-\varepsilon} \leq \psi(\mathbf{q})
$$

We define for $v>0$ the set

$$
\begin{aligned}
& \mathcal{W}_{S}(m, n ; v)=\left\{A \in \operatorname{Mat}_{m \times n}\left(\mathbb{F}\left(\left(X^{-1}\right)\right)\right):\right. \\
& \left.\qquad\|\mathbf{q} A\|<|\mathbf{q}|_{\infty}^{-v} \text { for infinitely many } \mathbf{q} \in S\right\} .
\end{aligned}
$$

By the above, for any $\varepsilon>0$,

$$
\mathcal{W}_{S}(m, n ; \lambda(\psi)+\varepsilon) \subseteq \mathcal{W}_{S}(m, n ; \psi) .
$$

As $\varepsilon>0$ is arbitrary, it therefore suffices to study the sets $\mathcal{W}_{S}(m, n ; v)$ and prove the corresponding full measure result and lower bound on the Hausdorff dimension for this set.

2.3. Measures and counting lemmas. We will use some probabilistic lemmas to prove the second and third parts of the theorem. The method which 
we will use is adapted from that used by Dodson in [5]. First, we need some estimates for the measure and number of elements of various sets. We define for $\mathbf{q} \in S$ and $\varepsilon>0$ the set

$$
B(\mathbf{q}, \varepsilon)=\{A \in U:\|\mathbf{q} A\|<\varepsilon\} .
$$

From [8, equation (2.6)] we extract the following lemma:

Lemma 3. Let $\varepsilon, \varepsilon^{\prime}>0$ and let $m \geq 2$. Suppose that $\mathbf{q}, \mathbf{q}^{\prime} \in \mathbb{F}[X]^{m}$ are linearly independent over $\mathbb{F}\left(\left(X^{-1}\right)\right)$. Then

$$
\mu\left(B(\mathbf{q}, \varepsilon) \cap B\left(\mathbf{q}^{\prime}, \varepsilon^{\prime}\right)\right)=\mu(B(\mathbf{q}, \varepsilon)) \mu\left(B\left(\mathbf{q}^{\prime}, \varepsilon^{\prime}\right)\right) .
$$

Furthermore, from [8, equation (2.3)], we get

Lemma 4. For any $\mathbf{q} \in S$,

$$
\mu\left(B\left(\mathbf{q},|\mathbf{q}|_{\infty}^{-v}\right)\right) \asymp|\mathbf{q}|_{\infty}^{-v n} .
$$

In general, measuring the intersection of the sets defined in (9) is difficult. However, we may restrict ourselves to certain subsets for which the task is easier. When $m=1$, we define

$$
B^{\prime}(q, \varepsilon)=\left\{A \in U:|q A-\mathbf{p}|_{\infty}<\varepsilon \text { for }\left(q, p_{i}\right)=1 \text { for all } 1 \leq i \leq n\right\} .
$$

Here $(q, p)$ denotes the greatest common divisor of $q$ and $p$ in $\mathbb{F}[X]$, which is unique up to multiplication by an element in $\mathbb{F}$. We will also need an analogue of the Euler totient function, defined for any $q \in \mathbb{F}[X]$ to be

$$
\Phi(q)=\#\left\{q^{\prime} \in \mathbb{F}[X]:\left|q^{\prime}\right|<|q|, q^{\prime} \text { monic and }\left(q, q^{\prime}\right)=1\right\} .
$$

From [6, equation (9)], we distill the following lemma:

Lemma 5. For $\varepsilon>0$ small enough,

$$
\mu\left(B^{\prime}(q, \varepsilon)\right)=\varepsilon^{n} \frac{\Phi(q)^{n}}{|q|^{n}} .
$$

Additionally, we distill from [6, page 159] that

Lemma 6. For $q, q^{\prime} \in \mathbb{F}[X]$ and $\varepsilon, \varepsilon^{\prime}>0$,

$$
\mu\left(B^{\prime}(q, \varepsilon) \cap B^{\prime}\left(q^{\prime}, \varepsilon^{\prime}\right)\right) \ll \varepsilon^{n} \varepsilon^{\prime n} .
$$

We will now estimate the growth of the function $\Phi(q)$.

LEMMA 7.

$$
\Phi(q) \asymp|q| .
$$

Proof. The proof is simple. For the purposes of this proof, $\mu$ denotes the usual Möbius function. We use the fact that $\sum_{d \mid r} \mu(d)$ is zero whenever $r \neq 1$ 
and is one when $r=1$. Now, for $q \in \mathbb{F}[X]$ with $\operatorname{deg}(q)=n$,

$$
\begin{aligned}
\Phi(q) & =\sum_{\substack{\operatorname{deg}(p, q)=0 \\
p \text { monic } \\
|p|<|q|}} 1=\sum_{\substack{\operatorname{deg}(p, q)=r \\
p \text { monic } \\
|p|<|q|}} \sum_{d \mid r+1} \mu(d)=\sum_{d=1} \mu(d) \sum_{\substack{|p|=k^{n+1-d} \\
p \text { monic }}} 1 \\
& =\sum_{d=1}^{n} \mu(d) k^{n+1-d} \gg k^{n}=|q| .
\end{aligned}
$$

The $\gg$ follows because the preceding expression is a polynomial with leading term $k^{n}$. The converse upper inequality is trivial.

We will need the higher-dimensional analogue of the $\operatorname{sets} B^{\prime}(\mathbf{q}, \varepsilon)$ in the course of the proof. We will impose a further restriction on the resonant neighbourhoods in order to enable us to treat this situation. In particular, we need to control the measure of the overlaps when $\mathbf{q}$ and $\mathbf{q}^{\prime}$ are linearly dependent. For $\mathbf{q} \in \mathbb{F}[X]^{m}$ and $\varepsilon>0$, we define

$$
\begin{aligned}
B^{\prime \prime}(\mathbf{q}, \varepsilon)=\{A \in U: & |\mathbf{q} A-\mathbf{p}|_{\infty}<\varepsilon \\
& \text { for } \left.\left(\operatorname{gcd}\left(q_{1}, \ldots, q_{m}\right), p_{i}\right)=1 \text { for all } 1 \leq i \leq n\right\} .
\end{aligned}
$$

Here, $\operatorname{gcd}\left(q_{1}, \ldots, q_{m}\right)$ denotes the greatest common denominator of the coordinates of $\mathbf{q}$ in $\mathbb{F}[X]^{m}$, which is unique up to multiplication by an element of $\mathbb{F}$. For these sets, we also estimate the relevant measures:

LEMMA 8. For $\varepsilon>0$ small enough,

$$
\mu\left(B^{\prime \prime}(\mathbf{q}, \varepsilon)\right) \asymp \varepsilon^{n} .
$$

Proof. This is shown exactly as Lemma 5, by measuring each component of the set and summing over them. The asymptotic estimate follows on using Lemma 7.

Lemma 9. Let $\mathbf{q}, \mathbf{q}^{\prime} \in \mathbb{F}[X]^{m}$ with $\mathbf{q} \neq \mathbf{q}^{\prime}$ and let $\varepsilon, \varepsilon^{\prime}>0$ be small. Then

$$
\mu\left(B^{\prime \prime}(\mathbf{q}, \varepsilon) \cap B^{\prime \prime}\left(\mathbf{q}^{\prime}, \varepsilon^{\prime}\right)\right) \ll \varepsilon^{n} \varepsilon^{\prime n} \text {. }
$$

Proof. First,

$$
B^{\prime \prime}(\mathbf{q}, \varepsilon) \cap B^{\prime \prime}\left(\mathbf{q}^{\prime}, \varepsilon^{\prime}\right) \subseteq B(\mathbf{q}, \varepsilon) \cap B\left(\mathbf{q}^{\prime}, \varepsilon^{\prime}\right) .
$$

Hence, if $\mathbf{q}$ and $\mathbf{q}^{\prime}$ are linearly independent,

$$
\mu\left(B^{\prime \prime}(\mathbf{q}, \varepsilon) \cap B^{\prime \prime}\left(\mathbf{q}^{\prime}, \varepsilon^{\prime}\right)\right) \leq \mu\left(B(\mathbf{q}, \varepsilon) \cap B\left(\mathbf{q}^{\prime}, \varepsilon^{\prime}\right)\right) \asymp \varepsilon^{n} \varepsilon^{\prime n},
$$

by Lemmas 3 and 4 . Hence, we need only show the result when the vectors are linearly dependent. In this case, the result will follow if we can show that no overlap between the sets is "too large".

Let $\widehat{\mathbf{q}}$ be a primitive vector in the direction of $\mathbf{q}$, i.e., a polynomial vector such that the greatest common denominator of the coordinates is of absolute value 1 and such that there are $\lambda, \lambda^{\prime} \in \mathbb{F}[X]$ with $\mathbf{q}=\lambda \widehat{\mathbf{q}}$ and $\mathbf{q}^{\prime}=\lambda^{\prime} \widehat{\mathbf{q}}$. 
Suppose without loss of generality that $|\lambda| \geq\left|\lambda^{\prime}\right|$, and let $\mathbf{p}, \mathbf{p}^{\prime} \in \mathbb{F}[X]^{n}$ be fixed so that the coordinates of $\mathbf{p}$ (respectively those of $\mathbf{p}^{\prime}$ ) have greatest common denominator of absolute value 1 with $\lambda$ (respectively with $\lambda^{\prime}$ ). We define affine subspaces $H, H^{\prime}$ by

$$
\begin{aligned}
H & =H(\mathbf{q}, \mathbf{p})=\{A \in U: \mathbf{q} A=\mathbf{p}\}, \\
H^{\prime} & =H\left(\mathbf{q}^{\prime}, \mathbf{p}^{\prime}\right)=\left\{A^{\prime} \in U: \mathbf{q}^{\prime} A^{\prime}=\mathbf{p}^{\prime}\right\} .
\end{aligned}
$$

It is clear that $B^{\prime \prime}(\mathbf{q}, \varepsilon)$ is the union over $\varepsilon$-neighbourhoods of all such $H(\mathbf{q}, \mathbf{p})$ with $|\mathbf{p}|_{\infty} \leq|\mathbf{q}|_{\infty}$, where $\mathbf{p}$ satisfies the co-primality condition, and similarly for $B^{\prime \prime}\left(\mathbf{q}^{\prime}, \varepsilon^{\prime}\right)$.

To calculate the measure of the intersection, we first note that we may disregard contributions from components around such $H$ and $H^{\prime}$ if these are separated by a quantity $\geq \min \left\{\varepsilon /|\mathbf{q}|_{\infty}, \varepsilon^{\prime} /\left|\mathbf{q}^{\prime}\right|_{\infty}\right\}$ outside of a set of measure zero. We find an arithmetic condition implying this.

Let $A \in H, A^{\prime} \in H^{\prime}$. On taking the defining equations, we see that

$$
\lambda \lambda^{\prime} \widehat{\mathbf{q}}\left(A-A^{\prime}\right)=\lambda^{\prime} \mathbf{p}-\lambda \mathbf{p}^{\prime} .
$$

Consider the absolute value of each coordinate of the vector $\lambda \lambda^{\prime} \widehat{\mathbf{q}}\left(A-A^{\prime}\right)$ in turn. This is some expression of the form

$$
|\lambda|\left|\lambda^{\prime}\right|\left|\left(a_{1 j}-a_{1 j}^{\prime}\right) q_{1}+\cdots+\left(a_{m j}-a_{m j}^{\prime}\right) q_{m}\right| .
$$

If the absolute values of the individual summands have a unique maximum, this is the absolute value of the sum by (1). We show that these absolute values are different outside of a set of measure zero.

Suppose that two summands are of equal absolute value,

$$
\left|\left(a_{11}-a_{11}^{\prime}\right) q_{1}\right|=\left|\left(a_{12}-a_{12}^{\prime}\right) q_{2}\right|,
$$

say. Then there is an $\alpha \in \mathbb{F}$ such that

$$
\alpha\left(a_{11}-a_{11}^{\prime}\right) q_{1}=\left(a_{12}-a_{12}^{\prime}\right) q_{2},
$$

so that

$$
a_{11}-a_{12} \frac{q_{2}}{\alpha q_{1}}=a_{11}^{\prime}-a_{12}^{\prime} \frac{q_{2}}{\alpha q_{1}} .
$$

This shows that in order for this to happen, $\left(a_{11}, a_{12}\right)$ and $\left(a_{11}^{\prime}, a_{12}^{\prime}\right)$ must lie on some "line" whose "slope" is a rational function. Each such "line" defines an $(n m-1)$-dimensional affine space in $\mathbb{F}\left(\left(X^{-1}\right)\right)^{m n}$ and so a set of measure zero. There are only countably many of these, as there are only countably many rational functions over $\mathbb{F}$. Finally, there are only finitely many ways in which two summands in (11) can be of equal absolute value. Hence, the exceptional set is of measure zero, and we may disregard matrices falling within this set, $\mathcal{E}$ say.

Suppose now that $A, A^{\prime} \in U \backslash \mathcal{E}$. Then, for all $j \in\{1, \ldots, n\}$,

$$
|\lambda|\left|\lambda^{\prime}\right| \max _{1 \leq i \leq m}\left\{\left|\widehat{\mathbf{q}}_{i}\right|\left|a_{i j}-a_{i j}^{\prime}\right|\right\}=\left|\lambda^{\prime} p_{j}-\lambda p_{j}^{\prime}\right| .
$$


Suppose that for some $j_{0} \in\{1, \ldots, n\}$,

$$
\left|\lambda^{\prime} p_{j}-\lambda p_{j}^{\prime}\right| \geq \varepsilon\left|\lambda^{\prime}\right|
$$

It then follows that

$$
\begin{aligned}
\left|A-A^{\prime}\right|_{\infty} & =\max _{1 \leq i \leq m, 1 \leq j \leq n}\left\{\left|a_{i j}-a_{i j}^{\prime}\right|\right\} \geq \max _{1 \leq i \leq m}\left\{\left|a_{i j_{0}}-a_{i j_{0}}^{\prime}\right|\right\} \\
& \geq \varepsilon /|\lambda||\widehat{\mathbf{q}}|_{\infty} \geq \varepsilon /|\mathbf{q}|_{\infty} \geq \min \left\{\varepsilon /|\mathbf{q}|_{\infty}, \varepsilon^{\prime} /\left|\mathbf{q}^{\prime}\right|_{\infty}\right\}
\end{aligned}
$$

which is what was to be shown. By a simple counting argument, it then follows that

$$
\mu\left(B^{\prime \prime}(\mathbf{q}, \varepsilon) \cap B^{\prime \prime}\left(\mathbf{q}^{\prime}, \varepsilon^{\prime}\right)\right) \ll \min \left\{\varepsilon^{n} /|\mathbf{q}|_{\infty}^{n}, \varepsilon^{\prime n} /\left|\mathbf{q}^{\prime}\right|_{\infty}^{n}\right\} N\left(\mathbf{q}, \mathbf{q}^{\prime}\right),
$$

where

$$
\begin{aligned}
N\left(\mathbf{q}, \mathbf{q}^{\prime}\right)=\#\left\{\mathbf{p}, \mathbf{p}^{\prime} \in \mathbb{F}[X]^{m}:\right. & |\mathbf{p}|_{\infty} \leq|\mathbf{q}|_{\infty},\left|\mathbf{p}^{\prime}\right|_{\infty} \leq\left|\mathbf{q}^{\prime}\right|_{\infty}, \\
& \left(p_{j}, \lambda\right)=\left(p_{j}^{\prime}, \lambda^{\prime}\right)=1 \\
& \text { and } \left.\left|\lambda^{\prime} p_{j}-\lambda p_{j}^{\prime}\right|<\varepsilon\left|\lambda^{\prime}\right| \text { for all } j=1, \ldots, n\right\} .
\end{aligned}
$$

Arguing exactly as in [6, pp. 158-159] for each coordinate, we find that

$$
N\left(\mathbf{q}, \mathbf{q}^{\prime}\right) \leq\left(\left|\mathbf{q}^{\prime}\right|_{\infty} \varepsilon+|\mathbf{q}|_{\infty} \varepsilon^{\prime}\right)^{n}
$$

Inserting (13) into (12), we obtain the desired inequality.

Finally, we will construct a subset of $S$ which contains a lot of elements and which grows in a regular fashion. First, define for $N \in \mathbb{N}$ the $N$ th $k$-adic block of $S$ :

$$
S_{N}=\left\{\mathbf{q} \in S: k^{N} \leq|\mathbf{q}|_{\infty}<k^{N+1}\right\} .
$$

Lemma 10. Let $\delta>0$. For any $N_{0} \in \mathbb{N}$, there is an $N \geq N_{0}$ such that

$$
\# S_{N} \geq k^{N(v(S)-\delta)} \text {. }
$$

Proof. Suppose to the contrary that for some $N_{0} \in \mathbb{N}$,

$$
\# S_{N}<k^{N(v(S)-\delta)}
$$

whenever $N \geq N_{0}$. Then, for any fixed $\varepsilon<\delta$,

$$
\begin{aligned}
\sum_{\substack{\mathbf{q} \in S \\
|\mathbf{q}|_{\infty} \geq k^{N_{0}}}}|\mathbf{q}|_{\infty}^{-v(S)+\varepsilon} & =\sum_{N=N_{0}}^{\infty} \sum_{\mathbf{q} \in S_{N}}|\mathbf{q}|_{\infty}^{-v(S)+\varepsilon} \ll \sum_{N=N_{0}}^{\infty} k^{-N(v(S)-\varepsilon)} \# S_{N} \\
& <\sum_{N=N_{0}}^{\infty} k^{-N(v(S)-\varepsilon)+N(v(S)-\delta)}=\sum_{N=N_{0}}^{\infty}\left(k^{\varepsilon-\delta}\right)^{N}<\infty
\end{aligned}
$$

On the other hand, by definition,

$$
\sum_{\mathbf{q} \in S}|\mathbf{q}|_{\infty}^{-v(S)+\varepsilon}=\infty
$$

for any $\varepsilon>0$. 
Lemma 10 implies the existence of an increasing sequence of integers $\left\{N_{t}\right\}_{t=1}^{\infty}$ for which $S_{N_{t}}$ has many elements.

2.4. Probabilistic lemmas. We will define random variables which will be used to construct a ubiquitous system. First, suppose that $m=1$, let $\delta>0$ be arbitrary and let $\left\{N_{t}\right\}_{t=1}^{\infty}$ be an increasing sequence of integers such that Lemma 10 holds for each $N_{t}$. Finally, define the function

$$
\varrho\left(k^{N}\right)=k^{-N(v(S)-\delta) / n} \log N .
$$

For each $t \in \mathbb{N}$, we define random variables on $U$,

$$
\nu_{t}(A)=\sum_{q \in S_{N_{t}}} \chi_{B^{\prime}\left(q, \varrho\left(k^{N_{t}}\right)\right)}(A),
$$

where $\chi_{E}$ denotes the characteristic function of the set $E$. Clearly, these variables count the number of sets $B^{\prime}\left(q, \varrho\left(k^{N_{t}}\right)\right)$ with $q \in S_{N_{t}}$ which contain a given element $A \in U$. Consequently,

$$
\nu_{t}^{-1}(0)=U \backslash \bigcup_{q \in S_{N_{t}}} B^{\prime}\left(q, \varrho\left(k^{N_{t}}\right)\right) .
$$

We calculate the first and second moments of these random variables.

First, let $r \in \mathbb{Z}$ be such that $k^{r} \leq \varrho\left(k^{N_{t}}\right)<k^{r+1}$. This implies that

$$
B^{\prime}\left(q, \varrho\left(k^{N_{t}}\right)\right)=B^{\prime}\left(q, k^{r}\right) .
$$

We easily estimate the mean value by integrating:

$$
\begin{aligned}
\mathbb{E}\left(\nu_{t}\right) & =\int_{U} \sum_{q \in S_{N_{t}}} \chi_{B^{\prime}\left(q, \varrho\left(k^{N_{t}}\right)\right)}(X) d \mu(X) \\
& =\sum_{q \in S_{N_{t}}} \int_{U} \chi_{B^{\prime}\left(q, k^{r}\right)}(X) d \mu(X) \asymp \sum_{q \in S_{N_{t}}} k^{r n} \asymp \varrho\left(k^{N_{t}}\right)^{n} \# S_{N_{t}},
\end{aligned}
$$

by Lemmas 5 and 7 .

We now estimate the second moment using the pairwise quasi-independence property from Lemma 6 , the measure estimate from Lemma 5 and Lemma 7:

$$
\begin{aligned}
\mathbb{E}\left(\nu_{t}^{2}\right)= & \int_{U} \sum_{q \in S_{N_{t}}} \chi_{B^{\prime}\left(q, \varrho\left(k^{N_{t}}\right)\right)}(X)^{2} d \mu(X) \\
& +\int_{U} \sum_{\substack{q, q^{\prime} \in S_{N_{t}} \\
q \neq q^{\prime}}} \chi_{B^{\prime}\left(q, \varrho\left(k^{N_{t}}\right)\right)}(X) \chi_{B^{\prime}\left(q^{\prime}, \varrho\left(k^{N_{t}}\right)\right)}(X) d \mu(X) \\
\ll & \mathbb{E}\left(\nu_{t}\right)+\sum_{\substack{q, q^{\prime} \in S_{N_{t}} \\
q \neq q^{\prime}}} \varrho\left(k^{N_{t}}\right)^{n} \varrho\left(k^{N_{t}}\right)^{n} \leq \mathbb{E}\left(\nu_{t}\right)+\mathbb{E}\left(\nu_{t}\right)^{2} .
\end{aligned}
$$


By (15) and (16), the variance $\sigma_{t}^{2}$ of $\nu_{t}$ satisfies

$$
\sigma_{t}^{2}=\mathbb{E}\left(\nu_{t}^{2}\right)-\mathbb{E}\left(\nu_{t}\right)^{2} \leq \mathbb{E}\left(\nu_{t}\right) .
$$

This has the following consequence.

Lemma 11. With $\nu_{t}$ and $\varrho(N)$ as above,

$$
\mu\left(\nu_{t}^{-1}(0)\right) \leq \frac{1}{\mathbb{E}\left(\nu_{t}\right)} \rightarrow 0 \quad \text { as } t \rightarrow \infty .
$$

Proof. The proof is easy. We use an alternative characterisation of the variance:

$$
\begin{aligned}
\sigma_{t}^{2} & =\int_{U}\left(v_{t}(A)-\mathbb{E}\left(\nu_{t}\right)\right)^{2} d \mu(A) \\
& \geq \int_{\nu_{t}^{-1}(0)}\left(v_{t}(A)-\mathbb{E}\left(\nu_{t}\right)\right)^{2} d \mu(A)=\mathbb{E}\left(\nu_{t}\right)^{2} \mu\left(\nu_{t}^{-1}(0)\right) .
\end{aligned}
$$

Hence,

$$
\mu\left(\nu_{t}^{-1}(0)\right) \leq \frac{\sigma_{t}^{2}}{\mathbb{E}\left(\nu_{t}\right)^{2}} \leq \frac{1}{\mathbb{E}\left(\nu_{t}\right)},
$$

by (17). Finally, note that by (15) and Lemma 10,

$$
\frac{1}{\mathbb{E}\left(\nu_{t}\right)} \asymp \frac{1}{\varrho\left(k^{N_{t}}\right)^{n} \# S_{N_{t}}} \ll \frac{1}{\left(\log N_{t}\right)^{n}} .
$$

As $N_{t}$ is increasing, this completes the proof.

We now proceed to discuss the case when $m \geq 2$. As before, we let $\delta>0$ be arbitrary and let $\left\{N_{t}\right\}_{t=1}^{\infty}$ be an increasing sequence of integers such that Lemma 10 holds for each $N_{t}$. For each $t \in \mathbb{N}$, we define random variables on $U$,

$$
\widetilde{\nu}_{t}(A)=\sum_{q \in \widetilde{S}_{N_{t}}} \chi_{B^{\prime \prime}\left(q, \varrho\left(k^{N_{t}}\right)\right)}(A) .
$$

These may be interpreted as $\nu_{t}$ above.

Calculating the first and second moments may be done exactly as in (15) and (16) by using the measure estimates of Lemmas 8 and 9 , so that

$$
\mathbb{E}\left(\widetilde{\nu}_{t}\right) \asymp \varrho\left(k^{N_{t}}\right)^{n} \# S_{N_{t}}, \quad \mathbb{E}\left(\widetilde{\nu}_{t}^{2}\right) \leq \mathbb{E}\left(\widetilde{\nu}_{t}\right)+\mathbb{E}\left(\widetilde{\nu}_{t}\right)^{2} .
$$

We recognise this as the main ingredients in the proof of Lemma 11, so that we immediately obtain the analogous version for $m \geq 2$.

Lemma 12. With $\widetilde{\nu}_{t}$ and $\varrho(N)$ as above,

$$
\mu\left(\widetilde{\nu}_{t}^{-1}(0)\right) \leq \frac{1}{\mathbb{E}\left(\widetilde{\nu}_{t}\right)} \rightarrow 0 \quad \text { as } t \rightarrow \infty
$$


2.5. Completing the proof. We first show (i), so suppose that $n v<v(S)$. The following result is classical: If $\left\{A_{i}\right\}$ is an infinite sequence of events in some probability space with probability measure $\mathbb{P}$, such that $\sum \mathbb{P}\left(A_{i}\right)=\infty$ and $A=\bigcap_{N=1}^{\infty} \bigcup_{i=N}^{\infty} A_{i}$, then

$$
\mathbb{P}(A) \geq \limsup _{N \rightarrow \infty} \frac{\left(\sum_{i=1}^{N} \mathbb{P}\left(A_{i}\right)\right)^{2}}{\sum_{i=1}^{N} \sum_{j=1}^{N} \mathbb{P}\left(A_{i} \cap A_{j}\right)} .
$$

We will apply this to the probability space $U$ equipped with the Haar measure and with the events $B^{\prime}\left(q,|q|^{-v}\right)\left(\operatorname{resp.} B^{\prime \prime}\left(\mathbf{q},|\mathbf{q}|_{\infty}^{-v}\right)\right)$.

First, let $m=1$ and fix $\delta<v(S)-n v$. By Lemmas 5 and 10,

$$
\sum_{q \in \mathbb{F}[X]} \mu\left(B^{\prime}\left(q,|q|^{-v}\right)\right) \gg \sum_{t=1}^{\infty} \sum_{q \in S_{N_{t}}}|q|^{-n v} \asymp \sum_{t=1}^{\infty} k^{N_{t}(v(S)-\delta-n v)}=\infty .
$$

Hence, (18) applies. Using Lemma 6, we find that

$$
\mu\left(\mathcal{W}_{S}^{*}\left(1, n ;|\cdot|^{-v}\right)\right) \geq c>0
$$

where $c$ comes from the right hand side of (18). Using Lemma 9 in place of Lemma 6 when $m \geq 2$, we find for all $m, n$ that

$$
\mu\left(\mathcal{W}_{S}^{*}\left(m, n ;|\cdot|_{\infty}^{-v}\right)\right) \geq c>0
$$

We now apply an inflation argument due to Cassels [3] to show that the measure must be full. This is in complete analogy with the proof of $[8$, Theorem 3]. In the above argument, we could just as easily have shown that $\mu\left(\mathcal{W}_{S}^{*}\left(m, n ;\left.\eta(\cdot)|\cdot|\right|_{\infty} ^{-v}\right)\right) \geq c>0$, where $\eta: \mathbb{F}[X]^{m} \rightarrow(0,1]$ is a function depending only on $|\mathbf{q}|_{\infty}$ which decreases to zero as $|\mathbf{q}|_{\infty}$ increases, such that $\lambda\left(\left.\eta(\cdot)|\cdot|\right|_{\infty} ^{-v}\right)=v$. Hence, we can find a point of metric density $A_{0} \in$ $\mathcal{W}_{S}^{*}\left(m, n ;\left.\eta(\cdot)|\cdot|\right|_{\infty} ^{-v}\right)$. Let $\varepsilon>0$. We may find an $r_{0} \in \mathbb{N}$ with

$$
\mu\left(\mathcal{W}_{S}^{*}\left(m, n ; \eta(\cdot)|\cdot|_{\infty}^{-v}\right) \cap B\left(A_{0}, k^{-r_{0}}\right)\right) \geq k^{-m n r_{0}}-\frac{\varepsilon}{k^{m n r_{0}}} .
$$

We scale the set by $X^{r_{0}}$ to obtain

$$
\mu\left(X^{r_{0}}\left(\mathcal{W}_{S}^{*}\left(m, n ; \eta(\cdot)|\cdot|_{\infty}^{-v}\right) \cap B\left(A_{0}, k^{-r_{0}}\right)\right)\right) \geq 1-\varepsilon,
$$

since $\mu\left(X^{r_{0}} B(c, r)\right)=k^{m n r_{0}} \mu(B(c, r))$ for any ball $B(c, r)$.

It follows that for any $\varepsilon>0$, we may find a set $T \subseteq U$ of measure $\mu(T) \geq 1-\varepsilon$ such that any $A \in T$ may be written in the form

$$
A=X^{r_{0}} A^{\prime}+P, \quad A^{\prime} \in \mathcal{W}_{S}^{*}\left(m, n ; \eta(\cdot)|\cdot|_{\infty}^{-v}\right), P \in \mathbb{F}[X]^{m n} .
$$

On considering the distance to the nearest polynomial vector for such elements, we find that $T \subseteq \mathcal{W}_{S}^{*}\left(m, n ;\left.k^{r_{0}} \eta(\cdot)|\cdot|\right|_{\infty} ^{-v}\right)$. Considering for a fixed element $A \in \mathcal{W}_{S}^{*}\left(m, n ;\left.k^{r_{0}} \eta(\cdot)|\cdot|\right|_{\infty} ^{-v}\right)$ the (infinitely many) $\mathbf{q} \in \mathbb{F}[X]^{m}$ for which $\eta(\mathbf{q})<k^{-r_{0}}$ and for which $|\mathbf{q} A|_{\infty}<k^{r_{0}} \eta(\mathbf{q})|\mathbf{q}|_{\infty}^{-v}$ shows that 


$$
T \subseteq \mathcal{W}_{S}^{*}\left(m, n ; k^{r_{0}} \eta(\cdot)|\cdot|_{\infty}^{-v}\right) \subseteq \mathcal{W}_{S}^{*}\left(m, n ;|\cdot|_{\infty}^{-v}\right) .
$$

This finishes the proof.

We now prove the lower bound on the dimension in (ii), which together with the upper bound found in $\S 2.1$ will complete the proof of the theorem. We will use the above estimates to construct a ubiquitous system of sets.

We define a function $\widetilde{\varrho}\left(k^{N}\right)=\varrho\left(k^{N}\right) k^{-N+1}$, where $\varrho$ is the function defined in (14), and sets

$$
\widetilde{B}(\mathbf{q}, \varepsilon)=\left\{A \in U: \operatorname{dist}\left(A, \bigcup_{\mathbf{p} \in \mathbb{F}[X]^{n}} H(\mathbf{q}, \mathbf{p})\right)<\varepsilon\right\},
$$

where $H(\mathbf{q}, \mathbf{p})=\{A \in U: \mathbf{q} A=\mathbf{p}\}$. We shall prove that when $\mathbf{q} \in S_{N_{t}}$, then

$$
B\left(\mathbf{q}, \varrho\left(k^{N_{t}}\right)\right) \subseteq \widetilde{B}\left(\mathbf{q}, \widetilde{\varrho}\left(k^{N_{t}}\right)\right) .
$$

But this easily follows as clearly,

$$
|\mathbf{q}|_{\infty} \operatorname{dist}(A, H(\mathbf{q}, \mathbf{p})) \leq|\mathbf{q} A-\mathbf{p}|_{\infty} .
$$

Choosing $\mathbf{p}$ so that $|\mathbf{q} A-\mathbf{p}|_{\infty}<\varrho\left(k^{N_{t}}\right)$, and noting that $k^{N_{t}-1} \leq|\mathbf{q}|_{\infty}$ as $\mathbf{q} \in S_{N_{t}}$, we have shown (21).

We now claim that the system $\left(\bigcup_{\mathbf{p} \in \mathbb{F}[X]^{n}} H(\mathbf{q}, \mathbf{p}),|\mathbf{q}|_{\infty}\right)$, where $\mathbf{q}$ runs over $\bigcup_{t=1}^{\infty} S_{N_{t}}$, is ubiquitous with respect to $\widetilde{\varrho}(N)$, i.e.,

$$
\lim _{t \rightarrow \infty} \mu\left(U \backslash \bigcup_{1 \leq|\mathbf{q}|_{\infty} \leq k^{N_{t}}} \widetilde{B}\left(\mathbf{q}, \widetilde{\varrho}\left(k^{N_{t}}\right)\right)\right)=0 .
$$

But this follows from (21), as

$$
\begin{aligned}
U \backslash \bigcup_{1 \leq|\mathbf{q}|_{\infty} \leq k^{N_{t}}} \widetilde{B}\left(\mathbf{q}, \widetilde{\varrho}\left(k^{N_{t}}\right)\right) & \subseteq U \backslash \bigcup_{\mathbf{q} \in S_{N_{t}}} B\left(\mathbf{q}, \varrho\left(k^{N_{t}}\right)\right) \\
& \subseteq \begin{cases}U \backslash \bigcup_{\mathbf{q} \in S_{N_{t}}} B^{\prime}\left(\mathbf{q}, \varrho\left(k^{N_{t}}\right)\right) & \text { for } m=1, \\
U \backslash \bigcup_{\mathbf{q} \in S_{N_{t}}} B^{\prime \prime}\left(\mathbf{q}, \varrho\left(k^{N_{t}}\right)\right) & \text { for } m \geq 2 .\end{cases}
\end{aligned}
$$

By Lemmas 11 and 12, the measure of the right hand side tends to zero as $t$ tends to infinity.

Now using the above, $[8$, Lemma 6$]$ implies that

$$
\begin{aligned}
\operatorname{dim}_{\mathrm{H}}\left(\mathcal{W}_{S}^{*}(m, n ; \psi)\right) \geq n(m-1)+n \limsup _{t \rightarrow \infty} \frac{\log _{k} \widetilde{\varrho}\left(k^{N_{t}}\right)}{\log _{k}\left(k^{N_{t}(-v-1)}\right)} \\
=n(m-1)+\limsup _{t \rightarrow \infty} \frac{-N_{t}(v(S)+n-\delta)-1+\log _{k} \log N_{t}}{N_{t}(-v-1)} \\
=n(m-1)+\frac{v(S)+n-\delta}{v+1} .
\end{aligned}
$$

As $\delta$ was arbitrary, this completes the proof of Theorem 1. 
3. Proof of Theorem 2. We essentially use Rynne's method [13]. First, note that if $\psi(\mathbf{q})>1$ for infinitely many $\mathbf{q} \in \mathbb{F}[X]^{m}$, the theorem is trivially true. Hence, there is no loss of generality in supposing that $\psi(\mathbf{q}) \leq 1$ for all $\mathbf{q} \in \mathbb{F}[X]^{m}$, as we may ignore the finitely many cases for which this happens. We will prove four lemmas, which will imply the theorem. First, we show that the upper bound on the dimension is the right one.

Lemma 13. With $n, m, \psi$ and $\eta(\psi)$ as above,

$$
\operatorname{dim}_{\mathbf{H}}(\mathcal{W}(m, n ; \psi)) \leq n(m-1)+\min \{\eta(\psi), n\} .
$$

Proof. This is identical to the covering argument of $\S 2.1$, where we replace the balls of the cover by balls of radius $\psi(\mathbf{q}) /|\mathbf{q}|_{\infty}$. This does not affect the number of balls needed, and we do not repeat the argument here.

In order to show that the lower bound holds, we define a number of quantities to be used in the proof. Let $\psi$ be a function as in the statement of Theorem 2, let $N \in \mathbb{N}$ and let $v>0$. We define

$$
\begin{aligned}
& C(N, v ; \psi)=\#\left\{\mathbf{q} \in \mathbb{F}[X]^{m}:|\mathbf{q}|_{\infty} \leq N, \psi(\mathbf{q}) \geq|\mathbf{q}|_{\infty}^{-v}\right\}, \\
& \gamma(v ; \psi)=\sup \left\{\gamma \in \mathbb{R}: \limsup _{N \rightarrow \infty} C(N, v ; \psi) N^{-\gamma}>0\right\}, \\
& \text { defined whenever } \lim _{N \rightarrow \infty} C(N, v ; \psi)=\infty \text {, }
\end{aligned}
$$

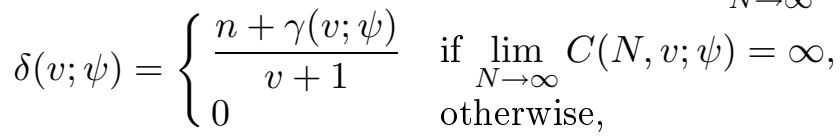

$$
\begin{aligned}
& \delta(\psi)=\sup _{v \geq 0} \delta(v ; \psi) .
\end{aligned}
$$

For arbitrary infinite subsets $S \subseteq \mathbb{F}[X]^{m}$, we will need an additional two definitions:

$$
\begin{aligned}
C(N ; S) & =\#\left\{\mathbf{q} \in S:|\mathbf{q}|_{\infty} \leq N\right\}, \\
\gamma(S) & =\sup \left\{\gamma \in \mathbb{R}: \limsup _{N \rightarrow \infty} C(N ; S) N^{-\gamma}>0\right\} .
\end{aligned}
$$

We first show that the exponent $\gamma(S)$ coincides with the exponent $v(S)$ of Theorem 1.

Lemma 14. Let $S \subseteq \mathbb{F}[X]^{m}$ with $\# S=\infty$. Then $v(S)=\gamma(S)$.

Proof. Fix $\varepsilon>0$. Choose $N_{0}$ so that $C(N ; S) \leq N^{\gamma(S)+\varepsilon}$ for $N \geq N_{0}$ and let $v>\gamma(S)+\varepsilon$. Then

Hence, $v(S) \leq \gamma(S)$.

$$
\begin{aligned}
\sum_{\mathbf{q} \in Q}|\mathbf{q}|_{\infty}^{-v} & =\sum_{r=1}^{\infty} \sum_{k^{r-1} \leq|\mathbf{q}|_{\infty}<k^{r}}|\mathbf{q}|_{\infty}^{-v} \ll \sum_{r=1}^{\infty} C\left(k^{r} ; S\right) k^{-r v} \\
& \ll \sum_{r=1}^{\infty} k^{r(\gamma(S)+\varepsilon-v)}<\infty
\end{aligned}
$$


For the reverse inequality, let $\gamma<\gamma(S)$ and choose a sequence $N_{r}$ with

$$
C\left(N_{r} ; S\right) N_{r}^{-\gamma}>\varepsilon \quad \text { and } C\left(N_{r+1} ; S\right)>2 C\left(N_{r} ; S\right)
$$

for all $r \in \mathbb{N}$. Clearly this is possible, as $\# S=\infty$. Now,

$$
\begin{aligned}
\sum_{\mathbf{q} \in Q}|\mathbf{q}|_{\infty}^{-\gamma} & \geq \sum_{r=1}^{\infty} \sum_{N_{r} \leq|\mathbf{q}|_{\infty}<N_{r+1}}|\mathbf{q}|_{\infty}^{-\gamma} \geq \sum_{r=1}^{\infty}\left(C\left(N_{r+1} ; S\right)-C\left(N_{r} ; S\right)\right) N_{r+1}^{-\gamma} \\
& >\sum_{r=1}^{\infty} \frac{1}{2} C\left(N_{r+1} ; S\right) N_{r+1}^{-\gamma}=\frac{1}{2} \sum_{r=1}^{\infty} \varepsilon=\infty .
\end{aligned}
$$

Hence, $\gamma<v(S)$, so that $\gamma(S) \leq v(S)$.

LEMma 15. With $n, m, \psi$ and $\delta(\psi)$ as above,

$$
\operatorname{dim}_{\mathrm{H}}(\mathcal{W}(m, n ; \psi)) \geq n(m-1)+\min \{\delta(\psi), n\} .
$$

Proof. We will deduce this lemma from Theorem 1 . The set $\mathcal{W}(m, n ; \psi)$ contains $n(m-1)$-dimensional affine spaces, and so the dimension is at least $n(m-1)$. Furthermore, it is at most $m n$, so we may suppose that $0<\delta(\psi) \leq n$.

Let $0<\varepsilon<\delta(\psi)$ and fix $v_{0} \geq 0$ so that $\delta\left(v_{0} ; \psi\right)>\delta(\psi)-\varepsilon>0$. Define a set

$$
S=\left\{\mathbf{q} \in \mathbb{F}[X]^{m}: \psi(\mathbf{q}) \geq|\mathbf{q}|_{\infty}^{-v_{0}}\right\} .
$$

This set is infinite by choice of $v_{0}$. Also,

$$
\mathcal{W}_{S}\left(m, n ;|\cdot|_{\infty}^{-v_{0}}\right) \subseteq \mathcal{W}(m, n ; \psi) .
$$

Finally, $C\left(N, v_{0} ; \psi\right)=C(N ; S) \rightarrow \infty$ as $N$ tends to infinity, so that

$$
\gamma\left(v_{0} ; \psi\right)=\gamma(S)=v(S)
$$

by Lemma 14 .

We apply Theorem 1 to $\mathcal{W}_{S}\left(m, n ;|\cdot|_{\infty}^{-v_{0}}\right)$. In view of $(22)$ and (23),

$$
\begin{aligned}
\operatorname{dim}_{\mathrm{H}}(\mathcal{W}(m, n ; \psi)) & \geq n(m-1)+\frac{n+v(S)}{1+v_{0}}=n(m-1)+\delta\left(v_{0} ; \psi\right) \\
& >n(m-1)+\delta(\psi)-\varepsilon .
\end{aligned}
$$

As $\varepsilon$ was arbitrary, this completes the proof.

LEMma 16. With $n, m, \psi, \delta(\psi)$ and $\eta(\psi)$ as above,

$$
\min \{\delta(\psi), n\} \geq \min \{\eta(\psi), n\} .
$$

Proof. Let $\eta>\delta(\psi)$. We will show that $\eta(\psi) \leq \eta$, so that we must have $\eta(\psi) \leq \delta(\psi)$. In order to accomplish this, we will split the series

$$
\sum_{\mathbf{q} \in \mathbb{F}[X]^{m}}|\mathbf{q}|_{\infty}^{n}\left(\frac{\psi(\mathbf{q})}{|\mathbf{q}|_{\infty}}\right)^{\eta}
$$


up into finitely many components, each of which converges. This will imply the result.

First, let $\mu=(m+n) / \eta$. Consider the set

$$
S^{\prime}=\left\{\mathbf{q} \in \mathbb{F}[X]^{m}: \psi(\mathbf{q})<|\mathbf{q}|_{\infty}^{-\mu}\right\} .
$$

Then, by [8, equation (1.4)],

$$
\sum_{\mathbf{q} \in S^{\prime}}|\mathbf{q}|_{\infty}^{n}\left(\frac{\psi(\mathbf{q})}{|\mathbf{q}|_{\infty}}\right)^{\eta} \leq \sum_{\mathbf{q} \in \mathbb{F}[X]^{m}}|\mathbf{q}|_{\infty}^{n-(\mu+1) \eta} \ll \sum_{r=1}^{\infty} k^{r(m+n-(\mu+1) \eta)}<\infty .
$$

Now, fix a $\theta \in(0,1-\delta(\psi) / \eta)$. Let $N_{0}$ be such that $C(N ; S) \leq N^{\gamma(v ; \psi)+\varepsilon}$ for $N \geq N_{0}$. For any $v>0$, we define sets

$$
S(v, \theta)=\left\{\mathbf{q} \in \mathbb{F}[X]^{m}:|\mathbf{q}|_{\infty}^{-v} \leq \psi(\mathbf{q}) \leq|\mathbf{q}|_{\infty}^{-v+\theta}\right\} .
$$

Clearly, since we can cover the set $[0, \mu]$ by intervals of the form $[v-\theta, v]$ there is a finite set $V$ such that

$$
\mathbb{F}[X]^{m}=S^{\prime} \cup \bigcup_{v \in V} S(v, \theta),
$$

since $\psi$ is assumed to be bounded above by 1 . Choose such a finite set $V$ and let $0<\varepsilon<(\eta-\delta(\psi)) \min _{v \in V}\{v\}$. For any $v \in V$,

$$
\begin{aligned}
\sum_{\mathbf{q} \in S(v, \theta)}|\mathbf{q}|_{\infty}^{n}\left(\frac{\psi(\mathbf{q})}{|\mathbf{q}|_{\infty}}\right)^{\eta} & \leq \sum_{\mathbf{q} \in S(v, \theta)}|\mathbf{q}|_{\infty}^{n-(v+1-\theta) \eta} \\
& \ll \sum_{r=1}^{\infty} \sum_{\substack{k^{r-1} \leq|\mathbf{q}|_{\infty} \leq k^{r} \\
\mathbf{q} \in S(v, \theta)}} k^{r(n-(v+1-\theta) \eta)} \\
& \ll \sum_{r=1}^{\infty} k^{r(n-(v+1-\theta) \eta+\gamma(v, \psi)+\varepsilon)}
\end{aligned}
$$

Hence, to show that this series converges, it suffices to show that the exponent is negative. But this follows as

$$
\begin{aligned}
n-(v+1-\theta) \eta+\gamma(v, \psi)+\varepsilon & <n-v \eta-\delta(\psi)+((v+1) \delta(v, \psi)-n)+\varepsilon \\
& <(\delta(\psi)-\eta) v+\varepsilon,
\end{aligned}
$$

which is less than zero by choice of $\varepsilon$. Hence,

$$
\sum_{\mathbf{q} \in S(v, \theta)}|\mathbf{q}|_{\infty}^{n}\left(\frac{\psi(\mathbf{q})}{|\mathbf{q}|_{\infty}}\right)^{\eta}<\infty
$$

and the lemma follows by $(24)-(26)$.

We now complete the proof of Theorem 2 by noting that the upper bound follows immediately from Lemma 13 . The lower bound follows on inserting the result of Lemma 16 into Lemma 15. 
Acknowledgements. I thank Yann Bugeaud for pointing out the paper [7] by Inoue and Nakada, and an anonymous referee for suggesting substantial improvements on the original manuscript. Finally, I thank Department of Mathematical Sciences at the University of Aarhus for their kind hospitality.

\section{References}

[1] V. I. Bernik and M. M. Dodson, Metric Diophantine Approximation on Manifolds, Cambridge Tracts in Math. 137, Cambridge Univ. Press, Cambridge, 1999.

[2] I. Borosh and A. S. Fraenkel, A generalization of Jarnik's theorem on Diophantine approximations, Nederl. Akad. Wetensch. Proc. Ser. A 75 = Indag. Math. 34 (1972), 193-201.

[3] J. W. S. Cassels, An Introduction to Diophantine Approximation, Cambridge Tracts in Math. Math. Phys. 45, Cambridge Univ. Press, New York, 1957.

[4] H. Dickinson and B. P. Rynne, Hausdorff dimension and a generalized form of simultaneous Diophantine approximation, Acta Arith. 93 (2000), 21-36.

[5] M. M. Dodson, Geometric and probabilistic ideas in the metric theory of Diophantine approximations, Uspekhi Mat. Nauk 48 (1993), no. 5 (293), 77-106 (in Russian); English transl.: Russian Math. Surveys 48 (1993), no. 5, 73-102.

[6] K. Inoue, The metric simultaneous Diophantine approximations over formal power series, J. Théor. Nombres Bordeaux 15 (2003), 151-161.

[7] K. Inoue and H. Nakada, On metric Diophantine approximation in positive characteristic, Acta Arith. 110 (2003), 205-218.

[8] S. Kristensen, On well-approximable matrices in a field of formal series, Math. Proc. Cambridge Philos. Soc. 135 (2003), 255-268.

[9] A. Lasjaunias, A survey of Diophantine approximation in fields of power series, Monatsh. Math. 130 (2000), 211-229.

[10] B. de Mathan, Approximations diophantiennes dans un corps local, Bull. Soc. Math. France Suppl. Mém. 21 (1970), 93 pp.

[11] A. D. Pollington and R. C. Vaughan, The k-dimensional Duffin and Schaeffer conjecture, Mathematika 37 (1990), 190-200.

[12] B. P. Rynne, The Hausdorff dimension of certain sets arising from Diophantine approximation by restricted sequences of integer vectors, Acta Arith. 61 (1992), 69-81.

[13] - The Hausdorff dimension of sets arising from Diophantine approximation with a general error function, J. Number Theory 71 (1998), 166-171.

[14] W. M. Schmidt, On continued fractions and Diophantine approximation in power series fields, Acta Arith. 95 (2000), 139-166.

[15] V. G. Sprindžuk, Metric Theory of Diophantine Approximations, Winston, Washington, DC, 1979.

School of Mathematics

The University of Edinburgh

James Clerk Maxwell Building

Kings Buildings, Mayfield Road

Edinburgh, EH9 3JZ, Scotland
Current address:

Department of Mathematical Sciences Faculty of Science, University of Aarhus Ny Munkegade, Building 530 DK-8000 Aarhus C, Denmark E-mail: sik@imf.au.dk 